\title{
Corrigendum: Assessing the function of STAS domain protein SypA in Vibrio fischeri using a comparative analysis
}

\author{
Cecilia M. Thompson and Karen L. Visick* \\ Department of Microbiology and Immunology, Loyola University Chicago, Maywood, IL, USA
}

Keywords: Vibrio fischeri, Vibrio parahaemolyticus, Vibrio vulnificus, biofilm formation, STAS domain proteins

\section{A corrigendum on}

Assessing the function of STAS domain protein SypA in Vibrio fischeri using a comparative analysis

by Thompson, C. M., and Visick, K. L. (2015). Front. Microbiol. 6:760. doi: 10.3389/fmicb.2015.00760

\section{OPEN ACCESS}

Edited and reviewed by: Yi-Cheng Sun,

Institute of Pathogen Biology

(CAMS-PUMC), China

${ }^{*}$ Correspondence:

Karen L. Visick

kvisick@luc.edu

Specialty section:

This article was submitted to

Food Microbiology,

a section of the journal

Frontiers in Microbiology

Received: 20 March 2017

Accepted: 10 April 2017

Published: 26 April 2017

Citation:

Thompson CM and Visick KL (2017)

Corrigendum: Assessing the function

of STAS domain protein SypA in Vibrio fischeri using a comparative analysis.

Front. Microbiol. 8:735.

doi: 10.3389/fmicb.2017.00735
Our work investigated the structure/function relationship of $V$. fischeri SypA using comparative analyses and mutagenesis approaches. We found that orthologs of SypA (RbdA, SypAVP) encoded by other Vibrio species ( $V$. vulnificus, $V$. parahaemolyticus) were able to complement the biofilm defect of a $V$. fischeri sypA mutant. These results indicated that the function of these proteins is conserved. We also identified and disrupted a set of conserved residues in SypA; a number of these mutations diminished or abolished SypA function in promoting biofilm formation. Our conclusions with respect to these studies remain intact.

However, we also reported results with respect to control of SypA by SypE. In subsequent experiments, we discovered that two of our strains were incorrect. This affects some of our results and conclusions with respect to the data shown in Figures 5, 7. In Figure 5, we reported that strains expressing $r b d A$ could largely overcome the inhibitory effect of SypE; however, we subsequently determined that this strain was incorrect as it did not express sypE. In the correct strain background, RbdA remains susceptible to control by SypE. Similarly, in Figure 7, we reported that a strain expressing a mutant form of SypA, SypA-R27A, was resistant to control by SypE, but that strain also proved to be incorrect as it did not express sypE. In the correct strain background SypA-R27A remains sensitive to SypE.

We have repeated the pertinent experiments with strains that express SypE, and now provide an updated, revised version of the text (and corresponding figures) as follows:

The corrected section entitled "SypA Proteins from Other Vibrios are Susceptible to Phosphorylation by V. fischeri SypE" should read as follows:

The three proteins each contain a stretch of highly conserved residues with a serine residue; in SypA, this serine (S56) is phosphorylated by SypE (Figure 1) (Morris and Visick, 2013a,b). Although sypE is missing from the chromosome of $V$. vulnificus and $V$. parahaemolyticus, it is possible that RbdA and SypA $A_{V P}$ are also controlled via phosphorylation and may retain the ability to interact with and be inactivated by SypE from $V$. fischeri. To determine if RbdA and SypA $A_{V P}$ were susceptible to inactivation by SypE, we expressed the sypA orthologs in a sypEcontaining sypA mutant $\left(\triangle s y p A s y p E^{+}\right)$and induced biofilm formation by overexpressing sypG. As expected, the negative control, a parent strain complemented with wild-type SypA fully susceptible to phosphorylation, failed to form wrinkled colonies, while the positive control, a parent strain 


\section{psypGIAsypA}
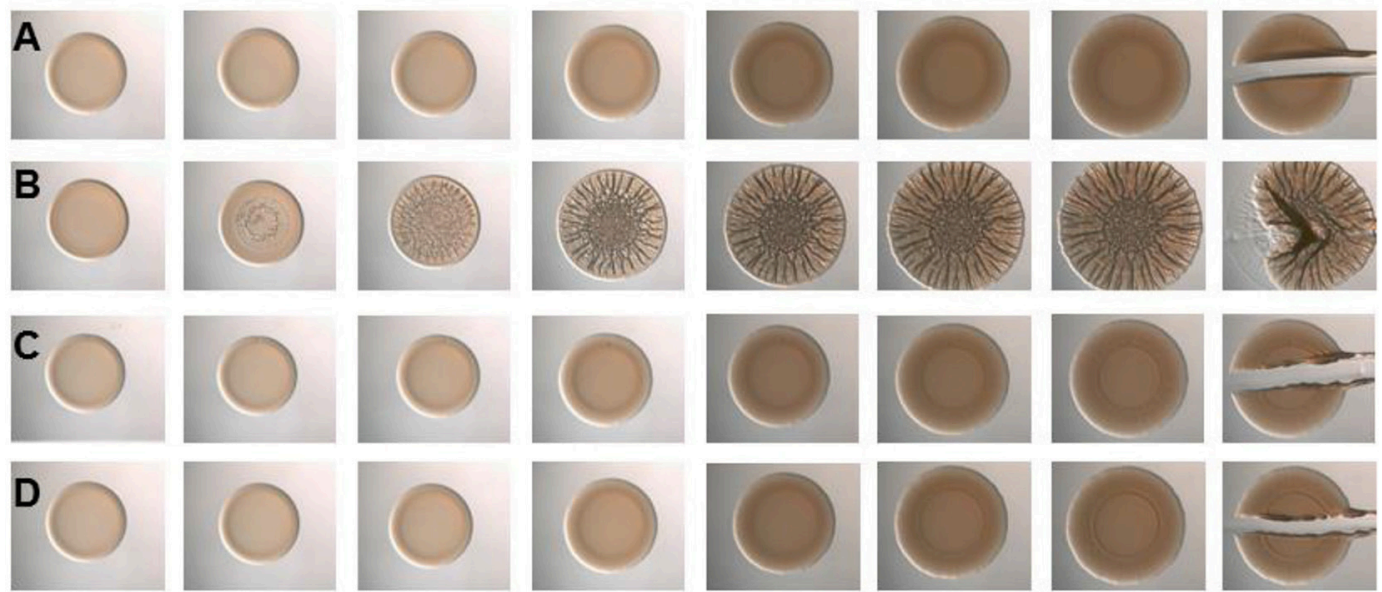

18.5

20

24

37.5

48

63.5
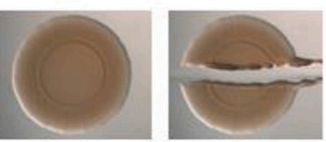

FIGURE 5 | SypA Proteins from Other Vibrios are Susceptible to Phosphorylation by $\boldsymbol{V}$. fischeri SypE. Development of colony morphology over time of sypG (pCLD56)-overexpressing derivatives of $\triangle$ sypA (sypE+) strains that contain (A) native sypA (KV5479), (B) sypA-S56A (expressing a mutant SypA that cannot be phosphorylated by SypE; KV5481), (C) rbdA (KV7309), or (D) sypAvp (KV7313). Cultures were spotted onto LBS plates containing tet, and the morphologies of the resulting colonies were assessed at the indicated times. Representative images are shown. At $72 \mathrm{~h}$, the colonies were disturbed with a toothpick to assess colony cohesiveness.

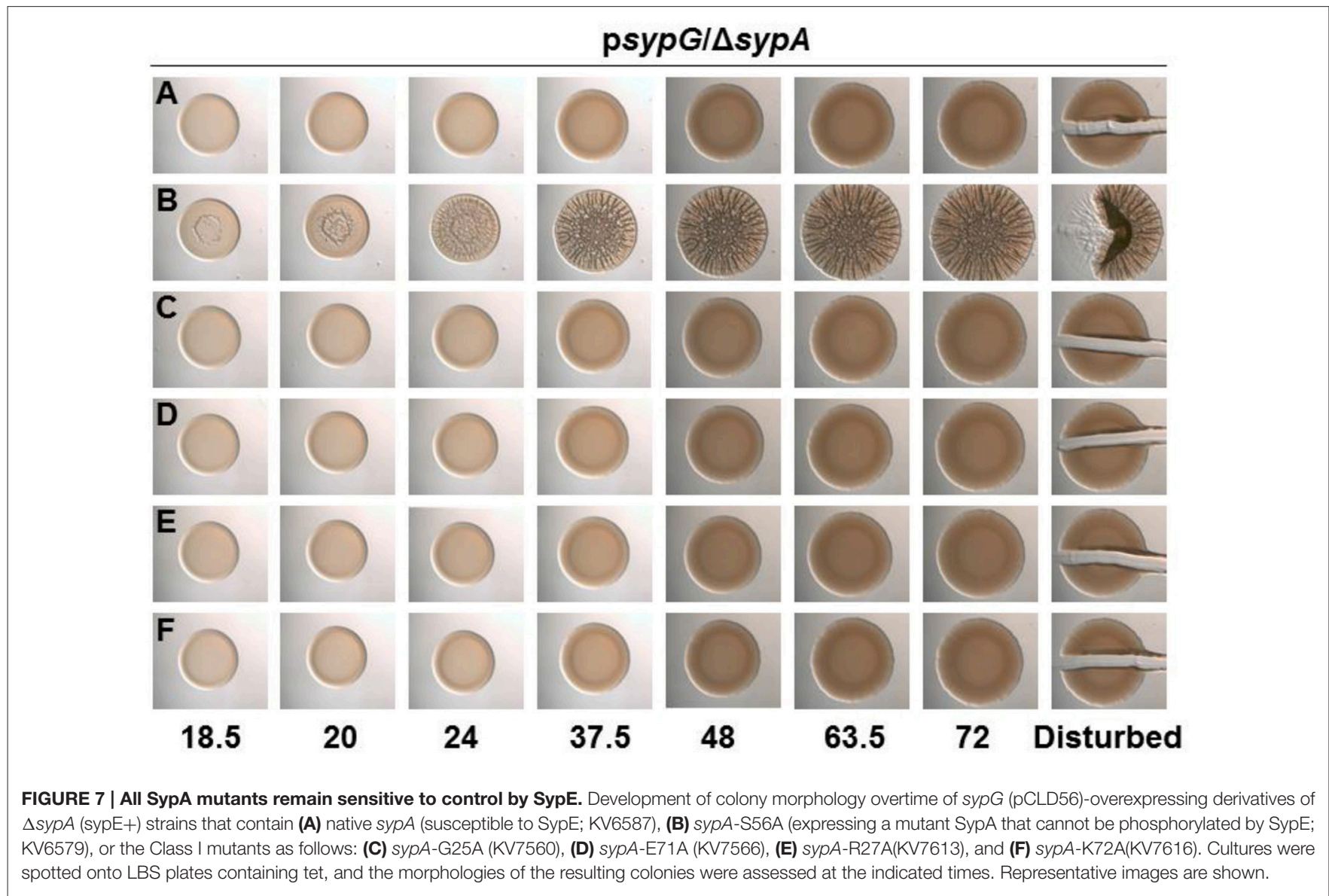


complemented with SypA ${ }^{\mathrm{S} 56 \mathrm{~A}}$, a mutant that cannot be inactivated via phosphorylation, formed robust wrinkled colonies in less than $24 \mathrm{~h}$ (Figures 5A,B). The colonies formed by the rbdA-containing strain failed to form wrinkled colonies, similar to the negative control. When we disrupted the colonies formed by $r b d A$-expressing $V$. fischeri, we found that the strain did not have cohesive properties, unlike the positive control (Figure 5C). Similarly, the syp $A_{\mathrm{VP}}$-expressing strain failed to form wrinkled or cohesive colonies even at later times (Figure 5D). These data indicate that SypA $A_{V P}$ and RbdA are both susceptible to regulatory control by $V$. fischeri SypE.

The corrected Figure 5 appears above.

The corrected section entitled "SypA Mutant is Resistant to Control by SypE" should have the following modified header and read as follows:

All SypA mutants remain sensitive to control by SypE. SypE binds to SypA and controls its activity via phosphorylation (Morris and Visick, 2013b). To date, Serine 56, the site of phosphorylation, is the only residue known to be critical for control by SypE. We hypothesized that other residues might facilitate the interaction between SypA and SypE, allowing for

\section{REFERENCES}

Morris, A. R., and Visick, K. L. (2013a). Inhibition of SypG-induced biofilms and host colonization by the negative regulator SypE in Vibrio fischeri. PLoS ONE 8:e60076. doi: 10.1371/journal.pone.00 60076

Morris, A. R., and Visick, K. L. (2013b). The response regulator SypE controls biofilm formation and colonization through phosphorylation of the sypencoded regulator SypA in Vibrio fischeri. Mol. Microbiol. 87, 509-525. doi: $10.1111 / \mathrm{mmi} .12109$ the phosphorylation of SypA, and that mutations in residues that facilitate this interaction would result in a SypA protein no longer recognized and/or phosphorylated by SypE. When introduced into a strain that expresses SypE, a mutant SypA that fails to interact with SypE will be "blind" to inhibition by SypE, resulting in biofilm formation under conditions in which it typically does not occur (e.g., sypG overexpression, Figure $\mathrm{S} 1 \mathrm{~B})$. We thus expressed the four Class I sypA mutant alleles [those able to promote wrinkled colony formation similar to the positive control (Figure 6C)] in a strain that contained $\operatorname{syp} E$ and induced biofilm formation by overexpressing sypG. As expected, the negative and positive control strains failed to form and formed, respectively, wrinkled colonies (Figures 7A,B). Not unexpectedly, all of the four mutants failed to induce wrinkled colony formation, indicating that the mutant SypA protein remained susceptible to inhibition by SypE (Figures 7C-F). We hypothesize that we may be unable to disrupt SypE's ability to control SypA by making single mutations. It is possible that multiple mutations may need to be made in SypA to prevent contact and phosphorylation by SypE.

The corrected Figure 7 appears above.

Conflict of Interest Statement: The authors declare that the research was conducted in the absence of any commercial or financial relationships that could be construed as a potential conflict of interest.

Copyright (c) 2017 Thompson and Visick. This is an open-access article distributed under the terms of the Creative Commons Attribution License (CC BY). The use, distribution or reproduction in other forums is permitted, provided the original author(s) or licensor are credited and that the original publication in this journal is cited, in accordance with accepted academic practice. No use, distribution or reproduction is permitted which does not comply with these terms. 